\title{
Entrelacs
}

ENTRELACS Cinéma et audiovisuel

$10 \mid 2013$

Le Toucher

\section{Du près au lointain, l'espace tangible du décor de cinéma}

\section{Grégory Bled}

\section{(2) OpenEdition}

\section{Journals}

\section{Édition électronique}

URL : http://journals.openedition.org/entrelacs/463

DOI : $10.4000 /$ entrelacs.463

ISSN : 2261-5482

Éditeur

Éditions Téraèdre

\section{Référence électronique}

Grégory Bled, « Du près au lointain, l'espace tangible du décor de cinéma », Entrelacs [En ligne], 10 | 2013, mis en ligne le 10 septembre 2013, consulté le 05 mai 2019. URL : http:// journals.openedition.org/entrelacs/463; DOI : 10.4000/entrelacs.463

Ce document a été généré automatiquement le 5 mai 2019.

Tous droits réservés 


\title{
Du près au lointain, l'espace tangible $d u$ décor de cinéma
}

\author{
Gregory Bled
}

1 Le proche et le lointain sont pour nous des représentations de distance et nous en déduisons une dimension temporelle. Conscients de notre corps en mouvement, nous pouvons exprimer une distance suivant un déplacement spatial naturel ou mécanique et cette distance peut alors donner lieu à une mesure du temps. Nous sommes capables de dire ce qui nous précède et ce que l'on précède, l'œil possède donc les clefs de l'espace. Il faut signaler, comme le fait Gérard Simon dans son livre Archéologie de la vision, que la vue en tant que sens possède des "sensibles propres " ${ }^{1}$; elle est seule sensible à la couleur, à la lumière. Par contre, elle possède avec le toucher des «sensibles communs» telles que la distance, la forme ou la grandeur. Ces « sensibles communs $»^{2}$ nous donnent la clef de ce qui peut être touché ou de ce qui nous paraît tactilement inaccessible. Les pleins et les vides nous parlent, la main les pressent, les devine et les ressent. Notre corps sera conscient de sa chute, aspiré par ce vide qui s'ouvre devant lui, puis conscient du futur choc sur cette surface qui se rapproche. Notre corps, notre main sont réceptifs aux creux et aux saillies, sensibles à ces zones de «Nahsicht » et de «Fernsicht $»^{3}$.

2 La localisation d'un corps dans l'espace ne se contente pas de coordonnées cartésiennes planaires $\mathrm{X}, \mathrm{Y}$. Une profondeur est nécessaire. Nous prendrons donc nos distances suivant des coordonnées cartésiennes tridimensionnelles, nous rajouterons un Z. Ces X, Y, Z, sont comme des chromosomes utiles à l'homme, ôtez-en un et l'homme devient l'image de luimême, sans épaisseur, sans corps physique.

3 Pour Allen Weiss, cette profondeur « existe parce que l'homme n'est pas Dieu $»^{4}$. Dieu est partout à la fois, il n'a donc pas besoin de se déplacer, il est là et déjà là-bas. MerleauPonty, expliquant les arguments de Berkeley, nous dit que la compréhension de la profondeur nécessite pour le sujet pensée et réflexion, il faut pour l'appréhender avoir ce regard latéral ${ }^{5}$. C'est en tant que décorateur et artiste peintre que je suis confronté à cette expression de l'espace, de l'espace de l'image à l'image de l'espace. Le décorateur et le peintre s'arrangent avec ces chromosomes. Le décorateur prend possession du lieu, 
l'occupe, le fractionne, le rejette, le réduit, le comprime. Le cadre de jeu du décorateur est le plateau de cinéma. Cet " espace clos illimité " ${ }^{6}$ n'est pas sans nous rappeler ce que Daniel Arasse nous dit de l'espace clos d'Alberti, théoricien de la perspective : « Alberti est un aristotélicien, il ne peut donc pas penser l'infini sur terre. Pour lui, l'espace est aristotélicien, c'est-à-dire clos et fait de la somme de ses lieux. Le point où se rejoignent les lignes de fuite n'est jamais appelé point de fuite, mais point central $»^{7}$. L'acteur peut pénétrer le décor implanté sur le plateau, y prendre ses marques, ses pas vont fouler le sol et ses mains vont toucher les murs, caresser les étoffes et les corps. Tout en prenant possession de ce lieu par la vue et le toucher, il le perdra. Notre vision nous interdit bien un regard à $360^{\circ}$. Notre corps ne peut pas être en contact avec tout ce qui nous cerne; il lui faudrait pour pouvoir toucher au même moment l'ensemble des corps et des objets qui l'entourent devenir gaz, air.

4 Si nous dépassons une chose par notre avancée spatiale et qu'elle échappe à notre vue, nous la soustrayons ; si notre main abandonne un corps, nous conservons dans le creux de la paume son souvenir. Nous ne perdons pas complètement cette chose; de matière, de substance, elle passe à l'état de fantôme, elle se fera mémoire; cette trace du souvenir participe à cette construction mentale qui nous donne toujours les clefs du lieu et de l'histoire.

Il suffit que je voie quelque chose pour savoir le rejoindre et l'atteindre, même si je ne sais pas comment cela se fait dans la machine nerveuse. Mon corps mobile compte au monde visible, en fait partie, et c'est pourquoi je peux le diriger dans le visible. Par ailleurs il est vrai que la vision est suspendue au mouvement. On ne voit que ce qu'on regarde. ${ }^{8}$

5 Ce « Nahsicht » est cette proximité à l'objet, ce gros plan dans lequel notre œil et notre main vont se perdre. La main caressant le plateau de bois ciré d'une table est dans l'ignorance même de l'objet table; la main parcourant la surface d'un cube ne peut réfléchir le cube «le cube à six faces égales est non seulement invisible, mais encore impensable; c'est le cube tel qu'il serait pour lui-même ; mais le cube n'est pas pour luimême, puisqu'il est un objet " ${ }^{9}$. Cet espace parcouru par la main échappe à ce "Fernsicht ", à la perspective. Il y aurait bien une différence entre ce près, ce premier plan sur cette étoffe soyeuse aux motifs de grandes fleurs, usée par le temps, qui habille la banquette sur laquelle sont assis Monsieur Neville et Madame Herbert et sa robe de dentelles fines, son chapeau de paille avec ruban de tulle volant au vent, et ce lointain qui s'ouvre sur ce jardin anglais, espace cadré par ce dispositif de treillis, dispositif largement illustré par Albrecht Dürer au début du XVIe siècle. Ce type d'installation aide à la mise en perspective dessinée de ce jardin mis en scène dans Meurtre dans un jardin anglais ${ }^{1}$. L'un sera visuellement touché et ressenti comme tel, il fera corps; l'autre nous restera tactilement abstrait, distant et fantomatique, allant jusqu'à l'effacement.

Le gros plan relève donc d'abord d'un régime physique, affectif, intense de l'image, d'une micro-physique des événements qui ne relève plus de la scène, du tableau, du théâtre, mais d'un espace ouvert, infini, fragmentaire, enfin libéré des mesures normatives de la perspective, de la profondeur de champ, du point de fuite. ${ }^{10}$

6 Le décorateur orchestrera près et lointain. Il suggérera à travers un champ visuel ${ }^{11}$ qui lui sera demandé par le réalisateur pour servir son histoire un assemblage de formes, de trajectoires et de matières. Le champ opératoire du décorateur sera comme ce templum antique où le destin des personnages sera fixé. Le champ mis à disposition par le « caillou » ${ }^{12}$, par l'objectif est bien ce qui nous révélera l'objet, et Merleau-Ponty nous dit : « quand je vois un objet, j'éprouve toujours qu'il y a encore de l'être au-delà de ce que je 
vois actuellement, non seulement de l'être visible, mais encore de l'être tangible ou saisissable par l'ouïe $»^{13}$. Il y a bien derrière cette fenêtre, au bout de la falaise, un monde qui se rattache par une surface, un tapis, un gaz à celui dans lequel je suis. Un lieu devance toujours un lieu. Ce monde est constitué d'un nombre incalculable de mondes tangibles dans lesquels je pourrais me mouvoir, me perdre. La surface, le tapis mis à la disposition du décorateur est le sol du plateau, les mondes qu'il proposera seront ou ne seront pas des espaces de jeu tactile pour les acteurs. Ce jeu de l'espace donnera naissance à des espaces de vide, des espaces intermédiaires. Et comme le montre l'artiste François Rouan dans ses œuvres de tressage, l'un est support à l'autre. François Rouan met en évidence des surfaces que l'on peut toucher par rapport à celles, invisibles qui échappent de ce fait au toucher. Pourtant ces surfaces cachées supportent, par le biais du tressage, les surfaces visibles et palpables. Le décorateur, contrairement à François Rouan, choisira une autre dimension, une autre profondeur et une autre hauteur. Pour échapper à la planéité, il prendra possession du plateau de cinéma, véritable palimpseste où le décor et l'histoire du film précédent ont été effacés, gommés pour céder la place à cet espace blanc immaculé qu'il va falloir habiter. A travers son travail spatial, il créera des lieux du jeu haptique et des lieux de jeu exclusivement optique.

7 Sur le plateau de cinéma, le décorateur partira toujours du rien, commencera dans du vide, dans cet espace "qui serait aussi des espaces flottants, indéterminés d'aucune spécificité particulière $»^{14}$. Ses constructions, ses objets structure vont s'implanter, créer des espaces de vide plus ou moins importants en fonction de la distance qui les sépare. Daniel Buren parle « d'objet-espace $»^{15}$ pour le vide et " d'objet-structure $»^{16}$ pour le plein. Il donne au vide (Vide : nom masculin, espace où il n'y a rien, Dictionnaire Quillet, 1948) une épaisseur; il devient objet (Objet: nom masculin, tout ce qui affecte les sens, Dictionnaire Quillet, 1948). Le vide devient une chose concrète perceptible et palpable, il prend du corps et nous livre ses trois dimensions, il répond à la vue et au toucher, il va au même titre que la structure prendre sa place dans l'espace, il sera acteur dans l'espace scénographique et nous révélera, même en échappant à notre regard, le temps, le son, la matière et l'odeur.

8 J'ai, il y a quelques années, tiré le portrait de cet espace rassemblé d'éléments hétérogènes qui devient homogène par ce tressage du visible et de l'invisible, dans le tableau l'Espace ${ }^{17}$ (Fig. 1). Ce décor qui ne peut évidemment pas être clos est obligatoirement ouvert et fermé, dehors et dedans, à la fois vide et plein ; « les opposés sont à tel point exclusifs qu'ils ne seraient l'un sans l'autre qu'abstraction, que la force de l'être s'appuie sur la faiblesse du néant qui est son complice... ${ }^{18}$. Le plein ne serait rien sans le vide, et il n'y a bien comme le dit Gaston Bachelard ${ }^{19}$ que la poésie qui puisse venir à notre aide pour définir ce qu'est cet espace; il est le ventre de la femme, il est ce cube avec ce sol en damier, ce pavement du Quattrocento, il est l'embrasure, l'ouverture sur la nature, il est le gardien des objets, il est le temple de la mémoire. « Votre maison est votre 
corps élargi $\aleph^{20}$. L'espace est bien notre corps élargi. L'espace est bien ce qui définit notre champ tactile.

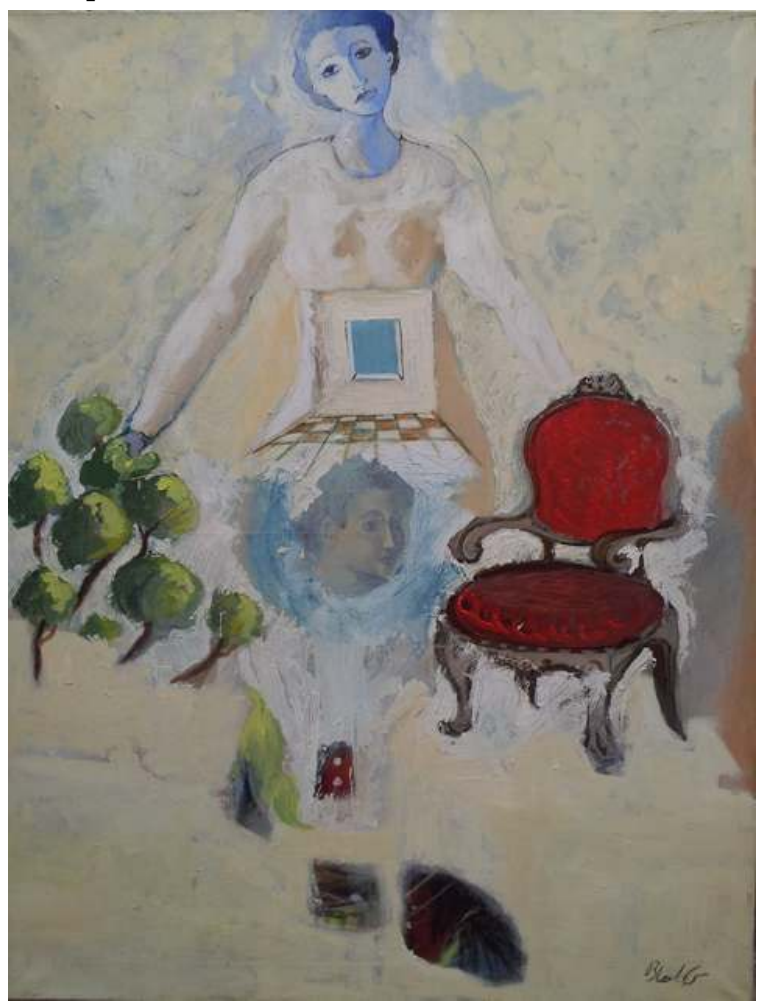

Fig. 1 : Grégory Bled, L'Espace, 2006, Peinture acrylique sur toile de $88 \times 116 \mathrm{~cm}$

\section{Commençons par le près}

Le jeudi 11 avril 2013, j'eus le grand plaisir de croiser M. Georges Lautner. Il était invité par la Cinémathèque de Toulouse pour les cinquante ans de son film Les Tontons flingueurs ${ }^{21}$. Je lui rappelai alors - lui évidemment ne s'en souvenait pas - que j'avais réalisé mon premier décor de film publicitaire pour lui. Il me donna, il y a presque 28 ans, ma première leçon de décor de cinéma. Les espaces créés sur le plateau représentaient des espaces de travail et il m'avait alors demandé de remplir les tiroirs du bureau d'accessoires divers (feuilles, crayons dossiers....) car quand l'acteur ouvrait un de ces tiroirs, il devait avoir quelque chose à manipuler, à palper. Dans ce lieu du décor à l'échelle humaine, contrairement au souhait de Georges Perec, il y a beaucoup plus que le Rien. Dans son livre Espèces d'espaces, l'écrivain est à la recherche d'un espace inutile : il lui arrive de penser à rien mais il n'arrive pas à penser le rien. «Comment penser le rien sans automatiquement mettre quelque chose autour de ce rien, ce qui en fait un trou, dans lequel on va s'empresser de mettre quelque chose, une pratique une fonction, un destin, un regard, un besoin, un manque, un surplus? $»^{22} C^{2}$ 'était bien cela que Georges Lautner me demandait : remplir ce rien, lui donner une histoire et lui réserver un destin.

L'espace du décor cinématographique est le lieu des souvenirs. Et la reconstitution de ces espaces doit, dans la mesure du possible, faire ressentir cette vie passée et devenir l'endroit du jeu présent pour l'acteur. Le stylisme et l'accessoirisation en sont les traces, la patine sur les murs en est le voile laissé par le temps, elle est la trace du vivant, de la crasse. 
Les Chinois ont un mot pour cela, "le lustre de la main "; les japonais disent

"l'usure" : le contact des mains au cours d'un long usage, leur frottement, toujours appliqué aux mêmes endroits, produit avec le temps une imprégnation grasse; en d'autres termes, ce lustre est donc bien la crasse des mains. ${ }^{23}$

11 Cette usure est le reflet du vieillissement de l'espace, elle est les rides du portrait spatial de cet autre acteur qu'est le décor. Cet acteur prendra lui aussi la lumière, il saura dissimuler ses atouts, il se fera «toko no ma », caché dans l'ombre ou se fera "shôji »" écrasé de lumière.

Le décor est la géographie de la vie de l'acteur, il constitue son ossature. Il est la coquille qui préserve les souvenirs que le décorateur, en accord avec le réalisateur, a souhaité lui offrir. On sait, comme le fait remarquer Georges Perec, que les espaces sont fragiles; ils changent et se transforment au cours de la vie :

Le temps va les user, va les détruire : rien ne ressemblera plus à ce qui était, mes souvenirs me trahiront, l'oubli s'infiltrera dans ma mémoire, je regarderai sans les reconnaître quelques photos jaunies aux bords tout cassés. Il n'y aura plus écrit en lettres de porcelaine blanche collées en arc de cercle sur la glace du petit café de la rue Coquillière "Ici, on consulte le Bottin" et" Casse-croûte à toute heure". ${ }^{25}$

Quelle chance d'avoir cette possibilité de créer ces espaces, de pouvoir recoller ces phrases sur la glace du petit café en lettres de porcelaine blanche, de se métamorphoser en architecte de l'espace et du temps, et de voir les personnages, les acteurs tourner les pages de ce bottin et chasser d'un mouvement de la main les miettes du casse-croûte de cette table en formica.

Le décorateur pourra par le jeu de matières ou de trompe l'œil donner à toucher du vrai et du faux. Daniel Arasse dans son livre le Détail ${ }^{26}$ nous parle de cette mouche, de cette représentation de la mouche devenant véritable trompe l'œil. Ce détail pictural qui a connu un véritable succès entre la moitié du Quattrocento et le milieu du XVIe siècle prend place sur le rebord du tableau ou sur la toile; ce détail est rejeté en dehors de la composition, il vient à la rencontre des spectateurs ${ }^{27}$. Ce détail ailé a ce double pouvoir de remettre la surface du tableau à sa place, la rendre encore plus plate, puis parfois dès qu'il s'intègre à la perspective spatiale du tableau, il peut en augmenter la tromperie. Il étire avec lui le détail architectural ou le corps sur lequel il est posé. Comme Cimabue, l'acteur pourra être trompé par les «Giotto du décor» qui maîtrisent matières et lumières. La lumière modelant le sujet peint lui procure ce volume, cette illusion de creux, de bosses, véritables paysages vallonnés. Les jeux d'ombres portées et propres soulignent cette profondeur, le noir d'une ombre fera creux, le blanc lumineux fera bosse. Il suffit de regarder ce trompe l'œil de bas-relief réalisé vers 1745 (Fig. 2) servant comme élément décoratif de haut de porte pour comprendre l'intérêt de cette maîtrise de la lumière et l'impact qu'elle peut avoir sur un volume. La qualité de ce trompe l'œil était telle que je me suis, à plusieurs reprises, placé latéralement aux panneaux peints, passant une main imaginaire sur leur surface, pour bien m'assurer de la planéité de l'œuvre. 


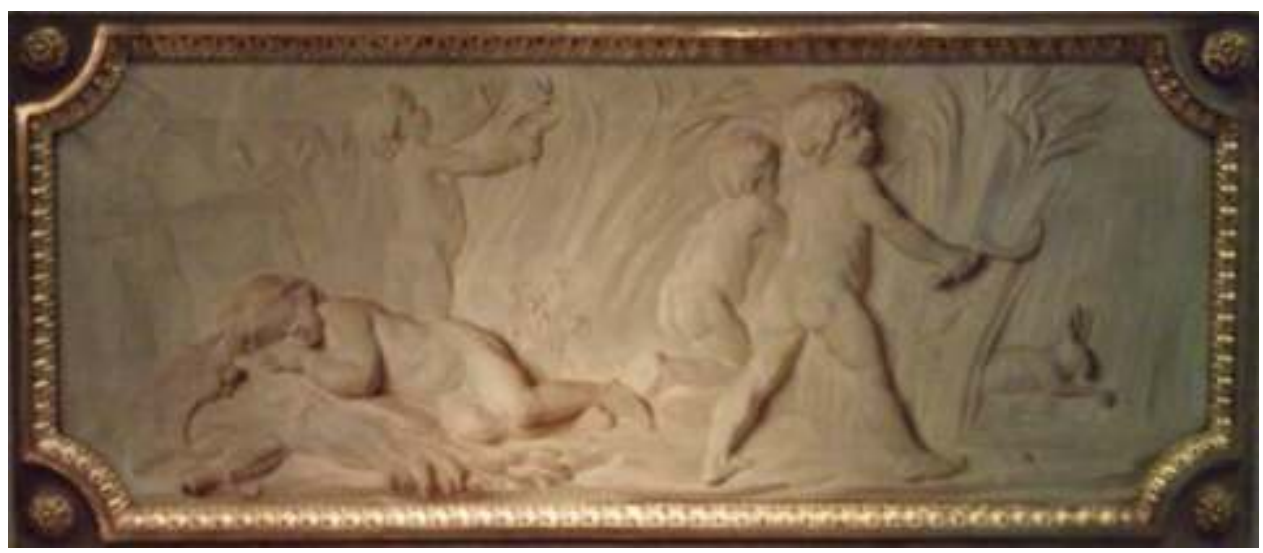

Fig. 2 : Huile sur toile, France, vers 1745, dessus de porte L'été, d'après les bas-reliefs des quatre saisons sculptés par Bouchardon pour la fontaine de la rue de Grenelle à Paris. Panneau présenté pour l'exposition sur le trompe l'œil au Musée des Arts Décoratif de Paris en 2013. Photo G. Bled.

Combien de fois avons-nous salivé devant des raisins gorgés de sucre, devant des poires juteuses se réfléchissant dans un gobelet d'argent ou une théière de porcelaine diaphane, comme dans les peintures de Chardin.

C'est la nature même. Les objets sont hors de la toile et d'une vérité à tromper les yeux (...) C'est que ce vase de porcelaine est de la porcelaine ; c'est que ces olives sont réellement séparées de l'œil par l'eau dans laquelle elles nagent ; c'est qu'il n'y a qu'à prendre ces biscuits et les manger; cette bigarade, l'ouvrir et la presser; ce verre de vin, et le boire ; ces fruits, et les peler ; ce pâté, et y mettre le couteau. ${ }^{28}$

Notre œil, notre "presque main »s'est parfois promené sur le grain subtil de la peau comme sur celle de La Vénus d'Urbin réalisée par Tiziano Vecelli dit le Titien ${ }^{29}$. Notre œil trompé avait déjà averti la main sur la façon de saisir la bigarade ou de caresser cette chair. L'œil du peintre Zeuxis, ${ }^{30}$ analysant la gestuelle de la main devant soulever l'étoffe qui dissimule le tableau de son ami peintre Parrhasios, s'aperçut que cette étoffe n'était pas réelle mais peinte. Nous sommes face à un « trompe-la main ». Cette information œilmain est ce que Gilles Deleuze nomme l'aspect digital dans les valeurs de la main.

Le digital semble marquer le maximum de subordination de la main à l'œil : la vision s'est faite intérieure, et la main est réduite au doigt, c'est-à-dire n'intervient plus que pour choisir les unités correspondant à des formes visuelles pures. Plus la main est ainsi subordonnée, plus la vue développe un espace optique" idéal", et tend à saisir ses formes suivant un code optique. ${ }^{31}$

18 Au cinéma, le trompe-l'œil a sa place dans l'espace; il se fait matière, et il peut en être la prolongation. Il doit se faire visible en tant que matière, et ressenti en tant que profondeur. Ce ressenti d'espace fonctionne s'il est imperceptible en tant que surface plane. Le trompe- l'œil est une image qui cherche à cacher sa nature d'image. "Le trompe-l'œil est ce qui, de la peinture, sollicite au premier chef le cinéma. C'est même ce qui, de la peinture, s'est intégré techniquement au cinéma depuis au moins Méliès. $»^{32} \mathrm{Le}$ trompe-l'œil spatial procède d'un travail sur les dimensions, il est le résultat d'une confiscation d'une dimension. De trois dimensions nous allons passer à deux, et c'est en cela que réside la magie car l'illusion intervient dans la simplification de l'espace. Le trompe-l'œil mérite une attention particulière. Il n'est plus objet, il en perd ses limites. Et il faudra au moment de l'étude de cette image, afin d'en modéliser l'espace, tenir compte de l'espace de jeu, l'espace tangible, car le mouvement cohabite mal avec le trompe-l'œil. Il faut en déterminer les zones dangereuses où il est aisé de basculer de cette maîtrise de 
la tromperie à la faute, au grotesque, à un effet mettant en doute la compréhension, la captation de l'espace. Le trompe-l'œil, lorsque celui-ci apparaît comme un mapping de l'espace du décor de cinéma, peut quant à lui subir toucher, caresses et coups.

Ce n'est pas une illusion de planches de bois que l'actrice Sabine Azéma vient toucher. Nous sommes en Angleterre, au cœur du Yorkshire, dans le village de Hutton Buscel où se trouve le jardin de Monsieur et Madame Teasdale. C'est sous le regard du chat en mosaïque qui trône au milieu de l'allée du jardin que Miles Coombes décide un jour de s'enfermer dans la remise au fond du jardin. Cette remise fabriquée de planches de bois brûlées par le soleil et surtout grisées et patinées par la pluie, avec son verrou et sa poignée rouillés, aux petites fenêtres fermées par un grillage à poule, sera le lieu de retraite de Miles. Pendant cinq semaines, il va y faire le point sur sa vie, ses déboires amoureux et ses envies cachées. Trois femmes, toutes trois jouées par Sabine Azéma, vont, à travers les planches, essayer de rentrer en contact avec Miles et nous allons alors assister à une palette de touchers, de coups, de caresses, de toqués, de heurts. Célia Teasdale, incapable d'avouer son amour secret à Miles, viendra lui amener ses repas, et, le front collé aux planches, essaiera de l'apercevoir à travers les joints. Sylvie Bell, employée chez les Teasdale, qui, mains à plat sur la porte, parle du voyage qu'ils doivent faire ensemble, toquera à la porte, puis se rappelant du poème que Miles lui a raconté, elle caressera les planches et arrachera à l'aide de ses ongles des fibres du bois. Puis Rowena Coombes, sa femme, viendra frapper ce mur de bois, le suppliant de sortir. Miles, voulant repartir à zéro, sortira en direction de la gare pour prendre le premier train, ou bien... Une autre décision, une autre histoire. ${ }^{33}$

Ce film, sans aucune scène d'intérieur, avec juste une amorce de l'entrée de la maison des Teasdale, de l'entrée de l'église, et de l'espace clos de la réserve, a été tourné sur les plateaux des studios d'Arpajon, en région parisienne. Travaillant à l'époque sur un autre plateau, j'ai eu le plaisir de déambuler à travers ces décors réalisés par Jacques Saulnier ${ }^{34}$. Lors de mon interview avec lui, en 2012, la discussion s'est portée à un moment donné sur cet espace du décor à l'échelle 1 . Ce lieu du jeu tactile où les acteurs laissent leurs empreintes diffère de ces lieux de la découverte et des lointains qui leur sont interdits. Ces espaces répondant à la loi de la perspective n'ont pas d'échelle en commun et le passage de l'un à l'autre nécessite un espace de transition, un espace intermédiaire.

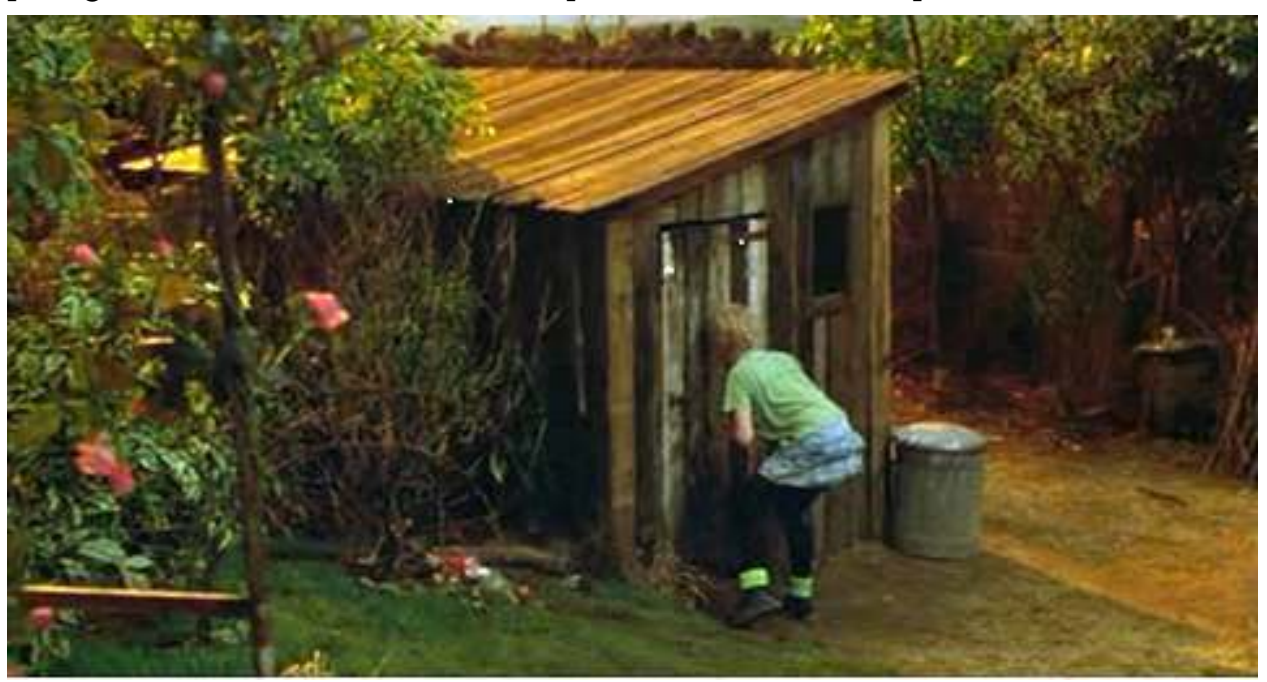

Fig. 3 : Smoking No Smoking, Alain Resnais (1993) @ Pyramide 


\section{L'espace intermédiaire}

21 Cet espace de transition est peut-être de l'ordre de l'éther, ce cinquième élément qui s'ajoute à la terre, à l'eau, au feu et à l'air.

En plus de ces quatre essences, Aristote voyait également le monde, comme plongé dans une quintessence, une cinquième essence, qu'il nomme éther. Le concept de vide tel que l'utilise aujourd'hui la physique semble assez proche de cette notion aristotélicienne d'éther. L'éther était pour Aristote le référent final, l'espace dans lequel tout était plongé et prenait consistance... Le vide quantique est devenu pour les physiciens le réservoir d'énergie dont les vibrations sont les particules, donc en quelque sorte la matière ${ }^{35}$.

Léonard de Vinci ${ }^{36}$, dans sa manière de traiter proche et lointain, de capter l'objet, le personnage en saillie par rapport au creux du paysage, a introduit et mis en évidence un espace qui sépare le dedans du dehors. Il veut maitriser la lisière qui les distingue, frontière qui souvent se résume au trait, au dessin, à ce linéament qui délimite les surfaces. Cette démarcation permet de décoller le premier plan du lointain. Le personnage utilise ce trait pour s'y détourer et ce trait devient pour Léonard de Vinci une zone qu'il faut atténuer, effacer. Il utilisera la méthode du sfumato et de l'évanescence. L'introduction de cette zone indécise et là, indécise peut être compris comme floue, mais aussi comme une hésitation à choisir son camp. Le trait ne sait plus s'il fait partie du personnage du premier plan ou du lointain, la frontière devient extrêmement brouillée. Le trait est comme ces zones de clair-obscur, sorte de déconstruction du rapport franc entre clair et obscur, dilution de la lumière dans l'ombre. Les nombreuses couches de glacis sont utilisées pour brouiller les pistes, la fumée qui entoure le personnage en diminue le coté préhensible, l'œil se perd un peu, hésite, espace creux ou saillie ? Léonard de Vinci élimine la masse des objets et des personnages qui nous semblent moins tactiles, mais d'un autre côté il augmente l'hapticité de l'air qui sépare les choses, l'hapticité de l'espace : on peut sentir la chaleur de l'air et le vent qui brouille au regard la mèche de cheveux. L'œil peut toucher cet espace intermédiaire, l'air prend du corps. Ce passage indécis entre optique et tactile est ce que Gilles Deleuze nomme l'haptique. ${ }^{37}$

Cette dilution que nous propose Léonard de Vinci dans ce passage entre près et lointain est ce franchissement d'une figuration à la forme. «On part d'une forme figurative, un diagramme intervient pour la brouiller, et il doit en sortir une forme d'une tout autre nature nommé figure $\aleph^{38}$. Ce passage n'est-il pas ce moment où, au cinéma, le changement de point nous fait basculer d'un corps à un autre, où dans la profondeur de champ, un objet perd de son espace tactile-optique, de sa figuration. Nous sommes dans une zone d'indiscernabilité, et c'est dans cette zone que l'œil touche.

Pour Léonard de Vinci, ce vide tout comme le vide quantique est source d'énergie, donc de matière, il possède cette épaisseur que l'on peut ressentir lorsque, pendant une promenade, on se rend compte qu'il y a beaucoup plus que le sol que l'on frappe des pieds, plus que les troncs d'arbres que l'on frôle des mains, plus que la douceur de l'herbe sur laquelle l'on se repose, il y a ce paysage qui se cache derrière la dune et qui vous est encore inconnu mais que de douces odeurs vous annoncent. Et le bruit des vagues encore invisibles vous remplit déjà l'œil, le vide est déjà lourd de promesses. Il y a la clairière cachée par ses arbres, il y a la lumière, il y a cet air gorgé d'embruns qui vous assèche la peau sous ce soleil blanc qui fait s'évaporer l'humidité de la terre chargée d'odeurs; votre peau se parfume de l'air qui vous enveloppe. 
Léonard de Vinci, par ses observations sur l'éloignement, la perspective de l'effacement, " porte par-là la perspective en un lieu où elle échappe aux mathématiques, et, surtout, il la détache en même temps du lien souverain qu'elle entretenait jusqu'alors avec l'idée de proportion. $»^{39}$ Nous échappons, au moment du changement de point dans cette zone indécise, aux règles de la géométrie. Les corps se perdent. Le décorateur en accord avec le chef opérateur, peut matérialiser une zone de voile, de cadres tendus de tulles, de grands vélums, mettre en place des dispositifs de rampe à gaz troublant l'air, utiliser des machines à fumée, à bruine. Tous ces agencements auront pour fonction première de brouiller, comme le dit Gilles Deleuze, le passage du près au lointain.

Une autre décision, une autre histoire...

Cette décision sera tragique pour Miles Coombes. Partis en voyage, Sylvie Bell, et lui se retrouvent au sommet d'une falaise, face à la mer. Une légère brume cache l'horizon, elle s'épaissit et engloutit une plate-forme pétrolière. Face à ce spectacle, Sylvie et Miles s'enlacent, se touchent et s'embrassent. La brume doucement envahit l'espace, les corps s'estompent, s'effacent et perdent de leur épaisseur tactile. Déjà le paysage n'existe plus, le lieu est brouillé, l'espace intermédiaire entre proche et lointain a pris possession de l'ensemble. Les personnages deviennent des formes puis des fantômes. Les corps, mains en avant se cherchent, il n'y a plus que la voix de Miles pour ramener Sylvie auprès de lui. Miles, perdu, désorienté, avalé par la brume tombera de la falaise.

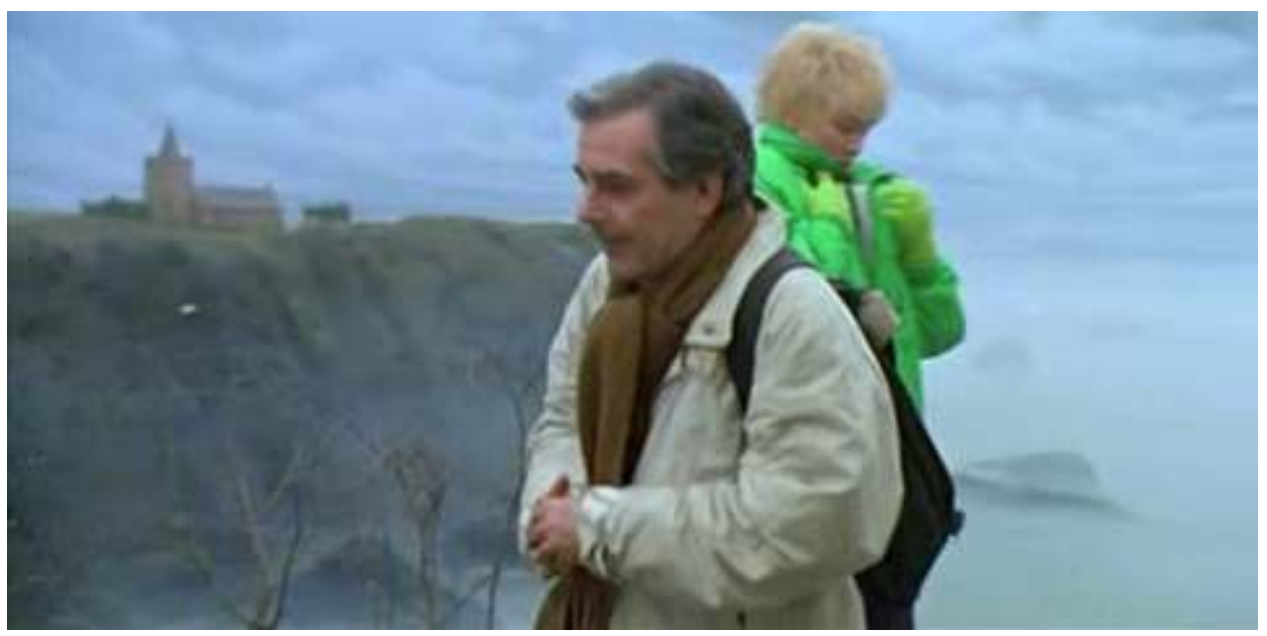

Fig. 4 : Smoking No Smoking, Alain Resnais (1993) @ Pyramide

\section{Les lointains et l'infini}

Jacques Saulnier me disait, lors de notre entretien, que pour donner une échelle à ces falaises du lointain, dans son décor de bord de mer de Smoking, No smoking, il y avait déposé une maquette d'église. Ce décorateur a été pendant un moment celui qui a eu le pouvoir d'approcher, de toucher ce presque infini. L'acteur n'a pas sa place dans ces espaces dans ces lointains, ils ne sont pour lui que des référents optiques. Pour le peintre décorateur, ces lointains deviennent l'endroit d'une liberté tactile, le geste peut s'y libérer ; la main en mouvement, dans une véritable insubordination peut y devancer la $v^{4} e^{40}$. Le décorateur fera en sorte dans son espace scénique de se rapprocher des lointains et de l'infini et comme l'évoque Gaston Bachelard, il aura l'imagination « miniaturante $»^{41}$. L'église de Jacques Saulnier « emmagasine de la grandeur. Elle est vaste à sa façon »" 


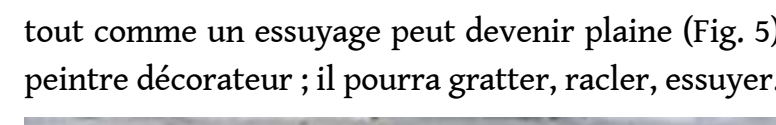

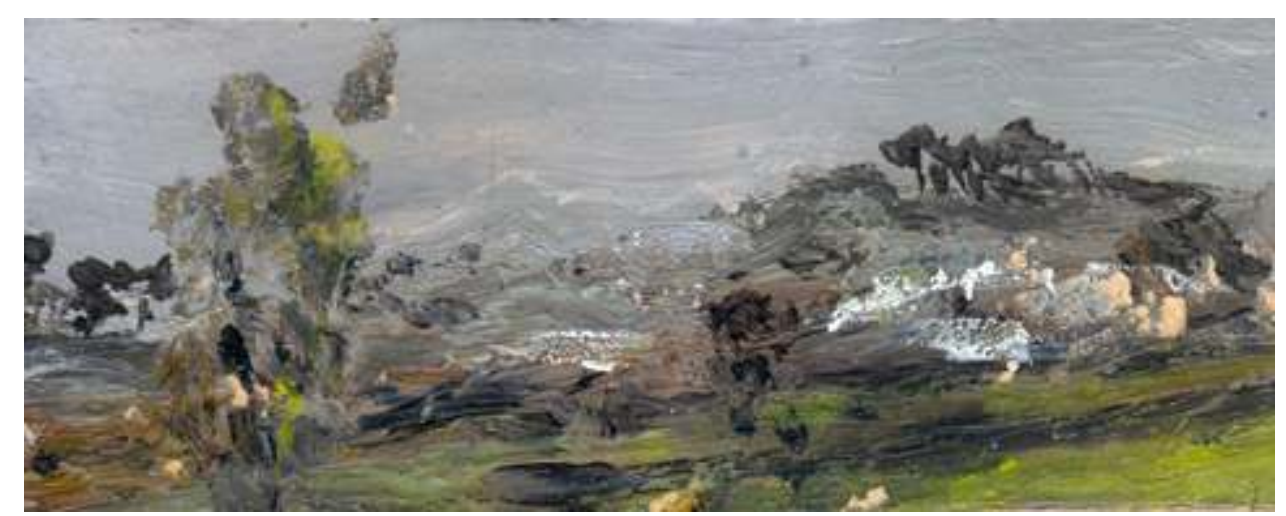

Fig. 5 : Grégory Bled, Paysage de lointain, 2012, Acrylique sur carton 5 × $12 \mathrm{~cm}$

L'abstraction n'est pas loin, c'est à travers une mise à distance de ce presque infini que le paysage peint ou modelé fera narration. Le décorateur, devenu géant par rapport à ses miniatures, peut d'un simple froissement de papier faire la montagne qui se diluera dans la lumière des projecteurs. Ce lointain, «fabrique d'ailleurs des miniatures en tous les points de l'horizon. Le rêveur, devant ces spectacles de la nature lointaine, détache ces miniatures comme autant de nids de solitude où il rêve de vivre ${ }^{44}$. Le lointain reste interdit au jeu de l'acteur, en s'en rapprochant, il nous dévoilerait ses failles, ses matières grossières, ses coups de pinceau sans finesse, il nous dévoilerait sa technique. Or, la technique ne doit surtout pas effriter la poésie. La proximité de cette peinture du lointain nous dévoile donc une épaisseur, une croûte, un paysage en relief. La caresse de notre main peut nous révéler un paysage de sillons, de bosses, de creux, véritable voyage en braille nous dévoilant le détail pictural que seul le peintre peut toucher. Pour avoir accès aux détails iconiques, il faudra prendre du recul, rejoindre Célia Teasdale et Miles Coombes dînant ensemble sur la terrasse, verre de cherry après verre de cherry, regardant rougir le ciel, tomber la nuit et au loin, le petit village se parer de lumières .

En peinture, le détail iconique se distingue du détail pictural qui, lui," ne fait pas image ; il ne représente pas et ne donne rien d'autre à voir que la matière picturale posée sur la toile, maniée et manipulée parfois jusqu'à en être triturée" ${ }^{\prime 4}$. Le détail iconique est donc lié aux concepts d'image, de représentation, de renvoi et de transparence ${ }^{46}$.

33 Il faudrait posséder un don divin pour représenter cet infini dont la caractéristique est d'être mouvant, d'être fugace, qui nous accompagne dans nos déplacements mais qui 
reste toujours à bonne distance, comme deux aimants qui se repoussent. Son indépendance lui donne un caractère mystérieux et pour certains, mystique, il pousse les hommes à dépasser cette chaîne de montagnes, connaître la fin de la plaine, descendre les rivières et traverser les océans.

L'infini c'est le divin. En ce sens, l'infini est le fondement des choses finies, le fini, c'est le limité, le divisible, le mesurable, le quantifiable. Le fini est susceptible d'accroissement et de diminution. L'infini ne peut être plus grand ou plus petit qu'il n'est. Il n'y a pas de plus ou de moins dans l'infini ${ }^{47}$.

Je ne sais pas si Dieu était présent au 14 Boulevard Raspail à Paris en janvier 2013, au lieu insolite, «The Museum of Everything » où lors de ma visite, j'ai découvert cet artiste qui, lui, a vu Dieu. Edmund Monsiel est né en Pologne en 1897. En 1942 chassé par les allemands, il se réfugie chez son frère. Il restera caché dans le grenier pendant 20 ans jusqu'à sa mort. Ce n'est qu'après sa mort que l'on y trouvera quelques 500 dessins. À travers son œuvre, Edmund Monsiel a voulu se rapprocher de l'infini, il voulait retrouver Dieu qui lui était apparu et, à l'aide d'un crayon, dessinait des visages, du plus grand au plus petit et jusqu'à son infiniment petit possible. Il nous faut utiliser une loupe pour rentrer dans son dessin; l'image prend alors une autre dimension, acquiert une profondeur et nous échappons à son cadre, nous nous y perdons mais là encore, l'artiste semble confronté à une contrainte technique, la grosseur de la mine de crayon qui lui interdit cet infini car le point le plus petit restera toujours un point.

Le décorateur, dans son espace mesurable, a la capacité de représenter en raccourci, l'ensemble du réel, «un coup de pinceau suffit à signaler une mer $»^{48}$ mais il reste incapable de s'approcher, d'effleurer réellement l'infini. Il fera lui aussi usage de cet adverbe presque et comme l'artiste Edmund Monsiel, il sera trahi par ses outils, par ce toucher, qui même s'il est frôlement, ne peut pas être aura. Le décorateur face à cet espace clos se heurtera aux murs du cyclorama qui seront ses limites, notre presque infini se fracassera contre. On peut rêver de les pénétrer, de les dépasser mais nous sommes bien dans un espace commensurable, il faudrait comme Alice pouvoir passer de l'autre côté.

\section{NOTES}

1. Gérard Simon, Archéologie de la vision, L'optique, le corps, la peinture, Paris : Seuil, 2003, p. 32p

2. Ibid.

3. Jacques Aumont, L'œil interminable, Paris, Nouvelles Éditions Séguier, 1995, p. 146. «Dans l'espace creux, quelque chose fait saillie (visuelle) : ce sont les objets, et notre vision connaît ainsi deux régimes, selon qu'elle a affaire à ce qui s'éloigne de nous et se creuse, l'espace, ou à ce qui pointe vers nous et s'offre, les objets. L'initiateur de ce fantasme, le sculpteur allemand Adolf Hildebrand, écrit carrément, en 1893, que"ces deux moyens de percevoir le même phénomène, non seulement ont une existence séparée dans nos facultés de la vue et du toucher, mais se retrouve unies dans l'œil" L'œil voit, mais aussi il touche : il y a dans la vision des perceptions optiques, purement visuelles, et des perceptions haptiques, visuelles-tactiles, double mode qui d'ailleurs répond à un autre partage, entre" Nahsicht" (la vue de près, la vision courante d'une 
forme dans l'espace vécu, où l'on peut s'approcher et toucher) et "Fernsicht" (La vue de loin, la vision de ces mêmes formes selon les lois spécifiques de l'art). »

4. Allen Weiss, Mirrors of infinity, Princeton Architectural Press, 1995, p. 34.

5. Maurice Merleau-Ponty, Phénoménologie de la perception, Paris, Éditions Gallimard, 1945. «Ce que j'appelle profondeur est en réalité une juxtaposition de points comparables à la largeur. Simplement, je suis mal placé pour la voir. Je la verrais si j'étais à la place d'un spectateur latéral, qui peut embrasser du regard la série des objets disposés devant moi, tandis que pour moi ils se cachent l'un l'autre. » Merleau-Ponty fera lui aussi référence à Dieu « Pour Dieu, qui est partout, la largeur est immédiatement équivalente à la profondeur», pp. 295-296.

6. Claude Régy, Espaces perdus, Besançon, Éditions Les Solitaires intempestifs, 1998. Claude Régy attribue cet espace clos illimité au théâtre, p. 25

7. Daniel Arasse, Histoires de peintures, Paris, Éditions Denoël, 2004, p. 45.

8. Maurice Merleau-Ponty, L'œil et l'esprit, Paris, Folio essais, 1964, pp. 16-17.

9. Maurice Merleau-Ponty, Phénoménologie de la perception, Paris, Éditions Gallimard, 1945, p. 236.

10. Pascal Bonitzer, Décadrages. Peintures et cinéma, Paris, Cahiers du cinéma Éditions de L'étoile, 1995 , p. 89.

11. Maurice Merleau-Ponty, Phénoménologie de la perception, Paris, Éditions Gallimard, 1945. Ce champ visuel est ce par quoi «... par position j'ai accès et ouverture à un système d'êtres, les êtres visibles, qu'ils sont à la disposition de mon regard.», pp. 250-251

12. J'ai le souvenir sur un tournage de Claude Zidi, de Monsieur Michel Serrault, avant une prise, déclamant son amour pour l'objectif : « Oh mon beau et gros caillou.... »

13. Maurice Merleau-Ponty, Phénoménologie de la perception, Paris, Éditions Gallimard, 1945, p. 250. 14. Claude Régy, Espaces perdus, Besançon, Éditions Les solitaires intempestifs, 1998, p. 135.

15. Entretien entre Daniel Buren et Pascale Marthine Tayou, «La Liberté de la contrainte», Revue L'officiel Art, avril mai juin 2012, p. 90.

16. Ibid.

17. Séries de peintures autour du livre de Khalil Gibran, Le Prophète. http://www.bledgpeintures.com/index.php?page=peintures\&categorie $=17$

18. Maurice Merleau-Ponty, Le Visible et l'invisible, Paris, Éditions Gallimard, 1964, p. 91.

19. Gaston Bachelard, La Poétique de l'espace, Paris, Presses universitaires de France, Bibliothèque de Philosophie Contemporaine, 1957, p. 25. En évoquant les souvenirs de la maison, nous additionnons des valeurs de songe; nous ne sommes jamais de vrais historiens, nous sommes toujours un peu poètes et notre émotion ne traduit peut-être que de la poésie perdue,

20. Khalil, Gibran, Le Prophète, Librairie Générale Française, 1993, p. 49.

21. Georges Lautner, Les Tontons flingueurs, France, Avec Lino Ventura, Bernard Blier, Francis Blanche, Jean Lefebvre. Dialogues Michel Audiard, 1963.

22. Georges Perec, Espèces d'espaces, Paris, Éditions Galilée, 1974/2000, p. 67.

23. Junichirô TanizaKi, Eloge de l'ombre, Traduit du japonais par René Sieffert, Éditions Verdier, 2011, p. 33.

24. Ibid.

25. Georges Perec, Espèces d'espaces, Paris, Éditions Galilée, 1974/2000, pp.179-180.

26. Daniel Arasse, Le Détail. Pour une histoire rapproché de la peinture, Paris, Flammarion, 1996, p. 117.

27. Daniel Arasse, Le Détail. Pour une histoire rapproché de la peinture, Paris, Flammarion, 1996, p. 117. Daniel Arasse cite une fausse anecdote rapportée par Vasari au sujet de Giotto : "Giotto, dans sa jeunesse, peignit un jour d'une manière si frappante une mouche sur le nez d'une figure commencé par Cimabue que ce maître, en se remettant à son travail, essaya plusieurs fois de la chasser avec la main avant de s'apercevoir de sa méprise. » 
28. Diderot, Essais sur la peinture, Salons de 1759, 1761, 1763, à propos du tableau Le Bocal d'olives de Chardin, Paris, Hermann, Éditeurs des Sciences et des Arts, Nouvelle édition, 2007, pp. 219-220.

29. http://www.univ-montp3.fr/pictura//GenerateurNotice.php?numnotice=A9179

30. E.H Gombrich, L'Art et l'illusion / psychologie de la représentation picturale, Phaidon Press Limited, 2002, pp. 172-173, nous cite le cas de l'anecdote contée par Pline. «Où l'on voit le peintre Parrhasios tromper son ami Zeuxis qui avait peint des grappes de raisin d'une si parfaite ressemblance que des oiseaux tentaient de les becqueter. Parrhasios invita son rival à entrer dans son atelier afin de regarder ses tableaux, et lorsque Zeuxis s'efforça de soulever une pièce d'étoffe qui semblait dissimuler un cadre, il s'aperçut qu'elle n'était pas réelle mais peinte : sur quoi il dut reconnaitre que Parrhasios l'avait emporté, car il ne s'était pas contenté de tromper des oiseaux privés d'intelligence, mais il avait abusé l'œil exercé d'un artiste. Envisagé à la lumière de la froide raison, ce triomphe de Parrhasios nous paraîtra un peu moins digne de susciter l'admiration. Toute l'expérience antérieure du malheureux Zeuxis devait lui indiquer qu'il n'y avait pas la moindre probabilité qu'il puisse se retrouver devant la peinture d'un voile. Quelques touches de lumière et d'ombre devaient suffire à lui faire « percevoir »le voile réel qu'il attendait, et d'autant mieux que son attention se fixait déjà sur l'attente de la phase suivante : le tableau qui allait lui être révélé. Les peintres de sujets trompe l'œil se sont souvent appuyés depuis lors sur les effets conjoints de l'attente et de l'illusion. »

31. Gilles Deleuze, Francis Bacon, Logique de la sensation, Paris, Éditions du Seuil, 2002, p. 145.

32. Bonitzer Pascal, Décadrages. Peintures et cinéma, Paris, Cahiers du cinéma Éditions de L'étoile, 1995, pp. 34-35. «Le trompe-l'œil serait-il le dénominateur commun du cinéma et de peinture? Du plan et du tableau ? On remarquera que si le cinéma a longtemps été méprisé comme art, c'est en raison de son caractère d'attraction illusionniste à l'usage des foules. De même la peinture a pu être condamnée pour la même raison, et plus particulièrement le trompe-l'œil a été dénoncé comme amusement mineur, tour de force d'escamoteur sans rapport avec la vraie peinture, avec la vérité en peinture. Depuis Baltrusaïtis cependant, le point de vue s'est renversé, et le trompel'œil tend à être replacé, comme dans les mythes antiques de Zeuxis et Parrhasios, aux confins, sinon au centre même de la peinture. Le trompe-l'œil est ce qui, de la peinture, sollicite au premier chef le cinéma. C'est même ce qui, de la peinture, s'est intégré techniquement au cinéma depuis au moins Méliès. Or, qu'est- ce qu'un trompe-l'œil ? Une représentation, qui se dénonce en deux temps comme l'illusion d'un spectacle réel, comme illusion de la réalité. La conscience et le tableau, le plan et le tableau si l'on veut, d'abord confondus dans l'illusion de réalité, se détachent brusquement l'un de l'autre. Et c'est bien ce détachement, non l'illusion, qui fait la jouissance du trompe-l'œil :" Qu'est- ce qui nous séduit et nous satisfait dans le trompe-l'œil ? demande Lacan. Quand est-ce qu'il nous captive et nous met en jubilation ? Au moment où par un simple déplacement de notre regard, nous pouvons nous apercevoir que la représentation ne bouge pas avec lui et qu'il n'y a là qu'un trompe-l'œil. Car il apparaît à ce moment-là comme autre chose qu'il se donnait, ou plutôt il se donne maintenant comme étant cet autre chose" »

33. Alain Resnais, Smoking, No Smoking, France, 1993, adapté de la pièce de Ayckbourn Alan, Intimate Exchanges, 1982.

34. Après une formation à l'école des Beaux-arts et à L'IDHEC, Jacques Saulnier sera l'assistant d'Alexandre Trauner et de Max Douy. A la fin des années 50, il devient chef décorateur, il travaillera entre autres pour Claude Chabrol, Louis Malle, Robert Hossein, Georges Clouzot...C'est à partir de L'Année dernière à Marienbad en 1960 que va naître une longue collaboration entre Jacques Saulnier et Alain Resnais.

35. Ludovic Bot, Philosophie des sciences de la matière, Paris, L'Harmattan, 2007, pp. 53-54.

36. Léonard De Vinci, Traité de la peinture, textes traduits et commentés par André Chastel, Éditions Calmann-Lévy, 2003. 
37. Gilles Deleuze, Francis Bacon, Logique de la sensation, Paris, Éditions du Seuil , 2002, p. 146. Chaque fois qu'il n'y aura plus subordination étroite dans un sens ou dans l'autre, ni subordination relâchée ou connexion virtuelle, mais quand la vue elle-même découvrira en soi une fonction de toucher qui lui est propre, et n'appartient qu'à elle, distincte de sa fonction optique.

38. Ibid.

39. Lucien Vinciguera, Archéologie de la perspective. Sur Piero della Francesca, Vinci et Dürer, Paris, PUF, p. 76.

40. Gilles Deleuze, Francis Bacon, Logique de la sensation, Paris, Éditions du Seuil, 2002. «On appellera manuel le rapport ainsi renversé.», p. 146.

41. Gaston Bachelard, La Poétique de l'espace, Paris, Presses universitaires de France, Bibliothèque de Philosophie Contemporaine, 1957, p. 141.

42. Gaston Bachelard, ibid. p. 195.

43. Léonard De Vinci, Traité de la peinture, textes traduits et commentés par André Chastel, Éditions Calmann-Lévy, 2003, p. 140.

44. Gaston Bachelard, La Poétique de l'espace, Paris, Presses universitaires de France, Bibliothèque de Philosophie Contemporaine, 1957, p. 159.

45. Daniel Arasse, Le Détail. Pour une histoire rapproché de la peinture, Paris, Flammarion, 1996, p. 268.

46. Klaus Speidel, L'Écriture du détail : allers retours entre peinture et littérature, dans « Complications de texte : les microlectures ", Fabula LHT (Littérature, histoire, théorie), n³ ; 01 septembre 2007.

47. Jean-Marie Nicolle, Jérôme Dokic, Philippe Drieux, René Lefebvre, (Textes réunis), Symboliques et Dynamiques de l'espace, Rouen, Publications de l'Université de Rouen, 2003, p. 57.

48. Emmanuelle Hénin, Ut Pictura Théatrum, Théâtre et peinture de la renaissance italienne au classicisme français, Genève, Travaux du Grand Siècle, Librairie Droz S.A, 2003, p. 316.

\section{RÉSUMÉS}

C'est en tant que décorateur et artiste peintre que je modèle l'espace, de l'espace de l'image à l'image de l'espace. L'espace du toucher, l'espace de jeu optique, l'espace de poésie selon Gaston Bachelard, l'espace sujet aux lois de la perspective, de celle d'Alberti à la perspective d'évanouissement de Léonard de Vinci, l'espace de jeu digital, tactile, haptique, manuel de Gilles Deleuze, sont les multiples facettes de l'espace du décor. Le cadre «clos illimité » de cet espace est le plateau de cinéma, ce lieu vide où le décorateur mettra en scène le présent de l'acteur sans en ignorer le passé et le futur. L'espace commensurable du décor de cinéma se conjugue du près au lointain. Le près est souvent l'espace du jeu, lieu du texte et de l'histoire, il situe l'espace tangible de l'acteur et possède l'échelle 1 . Dans ce lieu de la « feinte ", il est possible de toucher le réel et l'illusion, ce «trompe la main ». Le lointain échappe au toucher de l'acteur, mais il sera le lieu du toucher du décorateur. Le geste du peintre décorateur s'y libérera sans jamais pouvoir y reproduire l'infini. 


\section{AUTEUR}

\section{GREGORY BLED}

Professionnel associé à l'Ecole Supérieure d'Audiovisuel de Toulouse (ESAV), Gregory Bled est peintre et décorateur de cinéma. Il prépare actuellement une thèse ayant pour objet « l'espace vide comme élément constructif dans la poïétique du décor de cinéma ». Ses œuvres picturales sont en ligne sur le site : http://www.bledg-peintures.com 